\title{
Necessary and sufficient conditions for oscillation of first order neutral delay difference equations
}

\author{
A. Murugesan *, P. Sowmiya \\ Department of Mathematics, Government Arts College (Autonomous), Salem-636 007, Tamil Nadu, India \\ *Corresponding author E-mail: amurugesan3@gmail.com
}

Copyright (C)2015 A. Murugesan and P. Sowmiya. This is an open access article distributed under the Creative Commons Attribution License, which permits unrestricted use, distribution, and reproduction in any medium, provided the original work is properly cited.

\section{Abstract}

In this paper, we obtained some necessary and sufficient conditions for oscillation of all the solutions of the first order neutral delay difference equation with constant coefficients of the form

$$
\Delta[x(n)-p x(n-\tau)]+q x(n-\sigma)=0, \quad n \geq n_{0}
$$

by constructing several suitable auxiliary functions. Some examples are also given to illustrate our results.

Keywords: Oscillatory behavior, neutral, delay difference equation, constant coefficients.

\section{Introduction}

In this paper, we consider the first order neutral delay difference equation with constant coefficients of the form

$\Delta[x(n)-p x(n-\tau)]+q x(n-\sigma)=0, \quad n \geq n_{0}$,

where $n_{0}$ is a fixed nonnegative integer, $p$ and $q$ are positive real numbers, $\tau$ and $\sigma$ are positive integers and $\Delta$ is the forward difference operator defined by the equation $\Delta x(n)=x(n+1)-x(n)$.

Let $s=\max \{\tau, \sigma\}$ and $n_{0}$ be a fixed nonnegative integer. By a solution of (1), we mean a nontrivial real sequence $\{x(n)\}$ which is defined for all positive integer $n \geq n_{0}-s$ and satisfies (1) for $n \geq n_{0}$. A solution $\{x(n)\}$ of (1) is said to be oscillatory if for every positive integer $N>n_{0}$, there exists $n \geq N$ such that $x(n) x(n+1) \leq 0$, otherwise $\{x(n)\}$ is said to be nonoscillatory.

The theory of neutral delay difference equations presents complications and the results which are true for neutral difference equations may not be true for nonneutral difference equations. Besides its theoretical interest, the study of oscillatory behavior of solutions of neutral delay difference equations has some importance in applications.

The oscillation theory of neutral delay difference equation has been extensively developed during the past few years. See, for example, $[5,13,14,16]$ and the references cited therein. For the general background on difference equations, see the monographs $[2,4,7-9]$. 
G. Ladas [12] obtained a necessary and sufficient condition for oscillation of all solutions of the delay difference equation

$\Delta x(n)+q x(n-\sigma)=0, \quad n \geq n_{0}$

It was proved that all solutions of (2) are oscillatory if and only if the characteristic equation

$F(\lambda)=\lambda-1+q \lambda^{-\sigma}=0$

has no positive roots.

In [17], Zhou et al. established the necessary and sufficient conditions for oscillation of all solutions of Eq. (1) by investigating the nature of the roots of its characteristic equation.

Xiaohui Gong et al. [15] proved that all the solutions of the equation (1) are oscillatory if $0<p<1, \tau-\sigma>1$ and $q-\frac{\tau}{\tau-\sigma-1}\left(1-p^{\frac{1}{\tau}}\right)>0$.

Some oscillation results for neutral difference equations have given by Chen, Lalli and Yu [3], Georgiou, Grove and Ladas [6], and Lalli and Zhang [10,11]. In the special case of (homogenous) linear difference equations with constant coefficients, it is known that all solutions are oscillatory if and only if the associated characteristic equation has no positive roots.

The main aim of this paper is to establish a set of necessary and sufficient conditions for oscillation of all the solutions of (1) for the cases $0<p<1$ and $p>1$. Furthermore, we study deeply on oscillation of solutions under three cases $\tau<\sigma, \tau=\sigma$ and $\tau>\sigma$ when $p>1$. Besides that, we give some auxiliary criterions of main results (see Remarks 2.6, 2.15, 2.19 and 2.24), and obtain several simple sufficient conditions for oscillation of all the solutions of (1).

\section{Main results}

It is well-known from [1] that all solutions of (1) are oscillatory if and only if the characteristic equation of (1)

$f(\lambda) \equiv(\lambda-1)\left(1-p \lambda^{-\tau}\right)+q \lambda^{-\sigma}=0$

has no positive roots. It is not difficult to show that if $p=1$, then all the solutions of (1) are oscillatory.

Lemma 2.1 Let $0<p<1$. Then all the solutions of (1) are oscillatory if and only if the equation

$g(\mu)=\frac{q \mu^{\sigma+1}}{\mu-1}+p \mu^{\tau}-1=0$

has no positive roots in $\left(1,1+\frac{1}{\sigma}\right)$.

Proof. For $\lambda \geq 1$, we have that

$f(\lambda)=(\lambda-1)\left(1-p \lambda^{-\tau}\right)+q \lambda^{-\sigma} \geq q \lambda^{-\sigma}>0$.

Thus, any positive root of (4) must be in $(0,1)$.

Next, we consider the monotonicity of the function $g(\mu):=\frac{f\left(\frac{1}{\mu}\right)}{1-\frac{1}{\mu}}$. By direct calculation, it has that

$g^{\prime}(\mu)=\frac{\mu^{\sigma} \varphi(\mu)}{(\mu-1)^{2}}$,

where

$\varphi(\mu):=q[\sigma(\mu-1)-1]+p \tau(\mu-1)^{2} \mu^{\tau-\sigma-1}$.

Here, the function $\varphi(\mu)$ satisfies that following properties: 
(1) $\varphi(\mu)>0$ for $\mu \in\left(1+\frac{1}{\sigma},+\infty\right)$;

(2) $\varphi(\mu)$ is strictly increasing on $\left(1,1+\frac{1}{\sigma}\right)$ as the function $(\mu-1)^{2} \mu^{\tau-\sigma-1}$ is strictly increasing on $\left(1,1+\frac{1}{\sigma}\right)$.

Further,

$\varphi(1)=-q<0 \quad$ and $\quad \varphi\left(1+\frac{1}{\sigma}\right)=p \tau \frac{1}{\sigma^{2}}\left(\frac{\sigma+1}{\sigma}\right)^{\tau-\sigma-1}>0$.

Thus, we derive that the function $\varphi(\mu)$ has a unique zero $\theta$ on $\left(1,1+\frac{1}{\sigma}\right)$. Hence, $g^{\prime}(\mu)<0$ for $\mu \in(1, \theta)$ and $g^{\prime}(\mu)>0$ for $\mu \in(\theta,+\infty)$, which imply that $g(\mu)$ is decreasing on $(1, \theta)$ and increasing on $(\theta,+\infty)$. Therefore, $g(\mu)>0$ for $\mu \in(1,+\infty)$ if and only if (5) has no positive roots on $\left(1,1+\frac{1}{\sigma}\right)$.

By the above proof, it is not difficult to recognize that $g(\theta)$ is the minimum value of $g(\mu)$ on $\left(1,1+\frac{1}{\sigma}\right)$.

Lemma 2.2 Let $0<p<1$ and

$h(\mu):=q \mu^{\sigma+1}[(\tau-\sigma)(\mu-1)+1]-\tau(\mu-1)^{2}$.

Then all the solutions of (1) are oscillatory if and only if

$h(\theta)=q \theta^{\sigma+1}[(\tau-\sigma)(\theta-1)+1]-\tau(\theta-1)^{2}>0$,

where $\theta$ is a unique zero of the function $\varphi(\mu)$ that is defined by (7) on $\left(1,1+\frac{1}{\sigma}\right)$.

Proof. From the proof of Lemma 2.1, we have that $g(\mu)=0$ has no positive roots on $\left(1,1+\frac{1}{\sigma}\right)$ if and only if $g(\theta)>0$. Since

$g(\theta)=\frac{q \theta^{\sigma+1}}{\theta-1}+p \theta^{\tau}-1=\frac{h(\theta)}{\tau(\theta-1)^{2}}$,

we obtain Lemma 2.2 immediately.

Lemma 2.3 Let $0<p<1$. Then all the solutions of (1) are oscillatory if and only if one of the following conditions holds.

$\left(H_{1}\right) q \frac{(\sigma+1)^{\sigma+1}}{\sigma^{\sigma}} \geq 1 ;$

$\left(H_{2}\right) \bar{\theta}>\theta$, where $\theta$ and $\bar{\theta}$ are the unique zeros of $\varphi(\mu)$ and $h(\mu)$ (see (7) and (8)) on $\left(1,1+\frac{1}{\sigma}\right)$, respectively.

Proof. Let

$y(\mu)=\frac{h(\mu)}{(\mu-1)^{2}}=q \mu^{\sigma+1}\left(\frac{\tau-\sigma}{\mu-1}+\frac{1}{(\mu-1)^{2}}\right)-\tau$,

then

$y^{\prime}(\mu)=\frac{q \mu^{\sigma}}{(\mu-1)^{3}} z(\mu)$,

where

$z(\mu)=\sigma(\tau-\sigma)(\mu-1)^{2}+(2 \sigma-\tau)(\mu-1)-(\mu-1)-2$

which satisfies

$z(1)=-2<0 \quad$ and $\quad z\left(1+\frac{1}{\sigma}\right)=-\left(1+\frac{1}{\sigma}\right)<0$.

If $\tau \geq \sigma$, we get obviously that $z(\mu)<0$ for all $\mu \in\left(1,1+\frac{1}{\sigma}\right]$; If $\tau<\sigma$, we also get $z(\mu)<0$ for all $\mu \in\left(1,1+\frac{1}{\sigma}\right]$. Thus, $z(\mu)<0$ for all $\mu \in\left(1,1+\frac{1}{\sigma}\right]$ and from which and (11) mean that $y^{\prime}(\mu)<0$ for all $\mu \in\left(1,1+\frac{1}{\sigma}\right]$. Consequently, $y(\mu)$ is strictly decreasing on $\left(1,1+\frac{1}{\sigma}\right]$. Further,

$\lim _{\mu \rightarrow 1^{+}} y(\mu)=+\infty \quad$ and $\quad y\left(1+\frac{1}{\sigma}\right)=\left(\frac{q(\sigma+1)^{\sigma+1}}{\sigma^{\sigma}}-1\right) \tau$. 
Therefore, if $\frac{q(\sigma+1)^{\sigma+1}}{\sigma^{\sigma}} \geq 1$, then we have $y(\theta)>0$. Hence, $h(\theta)>0$. If $\frac{q(\sigma+1)^{\sigma+1}}{\sigma^{\sigma}}<1$, then we have $y\left(1+\frac{1}{\sigma}\right)<0$. Hence, it is easy to find that both functions $y(\mu)$ and $h(\mu)$ have an equal and unique zero $\bar{\theta}$ on $\left(1,1+\frac{1}{\sigma}\right)$. Consequently, $h(\theta)>0$ is equivalent to $\bar{\theta}>\theta$.

From Lemma 2.2, all the solutions of (1) are oscillatory if and only if one of $\left(H_{1}\right)$ or $\left(H_{2}\right)$ holds.

Corollary 2.4 If $p=0$, then all the solutions of (1) are oscillatory if and only if $\frac{q(\sigma+1)^{\sigma+1}}{\sigma^{\sigma}}>1$.

Theorem 2.5 Assume that $0<p<1$. Then all the solutions of (1) are oscillatory if and only if there exists a real number $\alpha \in\left(1,1+\frac{1}{\sigma}\right)$ such that

$\varphi(\alpha)=p \tau(\alpha-1)^{2} \alpha^{\tau-\sigma-1}+q[\sigma(\alpha-1)-1]>0$

$h(\alpha)=q \alpha^{\sigma+1}[(\tau-\sigma)(\alpha-1)+1]-\tau(\alpha-1)^{2}>0$.

Proof. From the proof of Lemma 2.1, the function $\varphi(\mu)$ has a unique zero $\theta$ on $\left(1,1+\frac{1}{\sigma}\right)$ and is strictly increasing on $\left(1,1+\frac{1}{\sigma}\right)$. If $\frac{q(\sigma+1)^{\sigma+1}}{\sigma^{\sigma}} \geq 1$, then from Lemma 2.3, all the solutions of (1) are oscillatory. Now, by the proof of Lemma 2.3, we know that $h(\mu)>0, \mu \in\left(1,1+\frac{1}{\sigma}\right)$. So that, conditions of the theorem hold.

If $\frac{q(\sigma+1)^{\sigma+1}}{\sigma^{\sigma}}<1$, then again from the proof of Lemma 2.3, the function $y(\mu)$ has a unique zero $\bar{\theta}$ on $\left(1,1+\frac{1}{\sigma}\right)$ and is strictly decreasing on $\left(1,1+\frac{1}{\sigma}\right)$. It can be seen that both functions $h(\mu)$ and $y(\mu)$ have the same sign in the interval $\left(1,1+\frac{1}{\sigma}\right)$. Hence, we obtain Theorem 2.5 from Lemma 2.3. The proof is complete.

Remark 2.6 The function $\varphi(\mu)$ has a unique zero $\theta$ on $\left(1,1+\frac{1}{\sigma}\right)$, and

$\varphi(\mu)<0$ for $\quad \mu \in(1, \theta)$

$\varphi(\mu)>0$ for $\mu \in\left(\theta, 1+\frac{1}{\sigma}\right)$

Further, we have that $\frac{q(\sigma+1)^{\sigma+1}}{\sigma^{\sigma}} \geq 1$ is equivalent to $h(\mu)>0, \mu \in\left(1,1+\frac{1}{\sigma}\right)$ and $\frac{q(\sigma+1)^{\sigma+1}}{\sigma^{\sigma}}<1$ is equivalent to the fact that $h(\mu)$ has a unique zero $\bar{\theta}$ on $\left(1,1+\frac{1}{\sigma}\right)$. Here,

$h(\mu)>0$ for $\quad \mu \in(1, \bar{\theta})$

$h(\mu)<0 \quad$ for $\quad \mu \in\left(\bar{\theta}, 1+\frac{1}{\sigma}\right)$

Thus, (1) has a non-oscillatory solution if and only if there exists a real number $\beta \in\left(1,1+\frac{1}{\sigma}\right)$ such that

$\varphi(\beta)=p \tau(\beta-1)^{2} \beta^{\tau-\sigma-1}+q[\sigma(\beta-1)-1] \leq 0$,

$h(\beta)=q \beta^{\sigma+1}[(\tau-\sigma)(\beta-1)+1]-\tau(\beta-1)^{2} \leq 0$.

Corollary 2.7 If there exists a real number $\alpha \in\left[\theta, 1+\frac{1}{\sigma}\right)$ such that

$q \alpha^{\sigma+1} \geq \sigma(\alpha-1)^{2}$

where

$\theta=1+\left[\frac{\frac{-q(\sigma+1)^{\sigma+1}}{\sigma^{\sigma}}+\sqrt{\left(\frac{q(\sigma+1)^{\sigma+1}}{\sigma^{\sigma}}\right)^{2}+4 p q \tau\left(\frac{\sigma+1}{\sigma}\right)^{\sigma+1}}}{2 p \tau}\right]$

for $p \in(0,1)$ and $\tau<\sigma$ hold, then all the solutions of (1) are oscillatory. 
Proof. Clearly, we have that $p \tau(\alpha-1)^{2}+q\left(\frac{\sigma+1}{\sigma}\right)^{\sigma+1}[\sigma(\alpha-1)-1] \geq 0$ for $\alpha \in\left[\theta, 1+\frac{1}{\sigma}\right)$ where

$\theta=1+\left[\frac{\frac{-q(\sigma+1)^{\sigma+1}}{\sigma^{\sigma}}+\sqrt{\left(\frac{q(\sigma+1)^{\sigma+1}}{\sigma^{\sigma}}\right)^{2}+4 p q \tau\left(\frac{\sigma+1}{\sigma}\right)^{\sigma+1}}}{2 p \tau}\right]$.

Therefore, it follows that

$$
\begin{aligned}
& \varphi(\alpha)=p \tau(\alpha-1)^{2} \alpha^{\tau-\sigma-1}+q[\sigma(\alpha-1)-1] \\
& >p \tau(\alpha-1)^{2}\left(\frac{\sigma}{\sigma+1}\right)^{\sigma+1}+q[\sigma(\alpha-1)-1] \geq 0, \\
& h(\alpha)=q \alpha^{\sigma+1}[(\tau-\sigma)(\alpha-1)+1]-\tau(\alpha-1)^{2} \\
& >\frac{\tau q \alpha^{\sigma+1}}{\sigma}-\tau(\alpha-1)^{2} \geq 0 .
\end{aligned}
$$

Therefore, the conditions of Theorem 2.5 hold.

Corollary 2.8 If $0<p<1$ and $\tau=\sigma$, then all the solutions of (1) are oscillatory if and only if there exists a real number $\alpha \in\left[\theta, 1+\frac{1}{\sigma}\right)$ such that

$h(\alpha)=q \alpha^{\tau+1}-\tau(\alpha-1)^{2}>0$,

where

$\theta=1+\left(\frac{-q(\tau+1)+\sqrt{q(\tau+1)[q(\tau+1)+4 p]}}{2 p \tau}\right)$.

Corollary 2.9 If $0<p<1, \tau>\sigma$ and there exists a real number $\alpha \in\left[\theta, 1+\frac{1}{\sigma}\right)$ where

$\theta=1+\frac{-q \sigma+\sqrt{q^{2} \sigma^{2}+4 p q \tau}}{2 p \tau}$

such that $q \alpha^{\sigma+1} \geq \tau(\alpha-1)^{2}$, then all the solutions of (1) are oscillatory.

Proof. Clearly, we have that $p \tau(\alpha-1)^{2}+q[\sigma(\alpha-1)-1] \geq 0$ for $\alpha \in\left[\theta, 1+\frac{1}{\sigma}\right)$ where

$\theta=1+\frac{-q \sigma+\sqrt{q^{2} \sigma^{2}+4 p q \tau}}{2 p \tau}$.

So that it follows that

$\varphi(\alpha)=p \tau(\alpha-1)^{2} \alpha^{\tau-\sigma-1}+q[\sigma(\alpha-1)-1]$

$\geq p \tau(\alpha-1)^{2}+q[\sigma(\alpha-1)-1] \geq 0$,

$h(\alpha)=q \alpha^{\sigma+1}[(\tau-\sigma)(\alpha-1)+1]-\tau(\alpha-1)^{2}$

$>q \alpha^{\sigma+1}-\tau(\alpha-1)^{2} \geq 0$.

If $\tau=\sigma+1$ and $\alpha=\theta$ then by Lemma 2.2, all the solutions of (1) are oscillatory. If either $\tau>\sigma+1$ or $\alpha>\theta$, then $\varphi(\alpha)>0$ and $h(\alpha)>0$. Therefore, the conditions of Theorem 2.5 hold.

So far, we have discussed and have obtained necessary and sufficient conditions for oscillation of all the solutions of (1) for $0<p<1$. Next, we will discuss the behavior of oscillation of solutions of (1) for $p>1$ under three subcases, namely, $\tau<\sigma, \tau=\sigma$ and $\tau>\sigma$.

Lemma 2.10 Let $p>1$. Then all the solutions of (1) are oscillatory if and only if the equation

$g(\mu)=\frac{q \mu^{\sigma+1}}{\mu-1}+p \mu^{\tau}-1=0$

has no positive roots on $\left(p^{-\frac{1}{\tau}}, 1\right)$. 
Proof. Since

$g(\mu)=\frac{q \mu^{\sigma+1}}{\mu-1}+p \mu^{\tau}-1>\frac{q}{\mu}+q \sigma+p-1$,

we know that $g(\mu)>0$ for $\mu \in(1, \infty)$. It is not difficult to see that $\frac{\mu^{\sigma+1}}{\mu-1}$ is strictly decreasing on $(0,1)$ while $\mu^{\sigma+1}$ is strictly increasing on $(0,1)$. Note that $p \mu^{\tau}-1=0$ at $\mu=p^{-\frac{1}{\tau}}$, we find that

$g(\mu)<0$ for $\quad \mu \in\left(0, p^{-\frac{1}{\tau}}\right]$.

Clearly, $f(1)=q>0$. Thus, $f(\lambda)$ has no positive roots which is equivalent to $g(\mu)$ has no positive roots on $\left(p^{-\frac{1}{\tau}}, 1\right)$.

Theorem 2.11 Suppose that $p>1$. Then all the solutions of (1) are oscillatory if and only if

$g(\mu)<0 \quad$ for $\quad \mu \in(0,1)$.

Lemma 2.12 Let $p>1$ and $\tau<\sigma$. Then all solutions of (1) are oscillatory if and only if

$h(\theta)=q \theta^{\sigma+1}[(\tau-\sigma)(\theta-1)+1]-\tau(\theta-1)^{2}<0$,

where $\theta$ is a unique zero of $(7)$ on $(0,1)$.

Proof. Firstly, we prove that $\varphi(\mu)$ has a unique zero $\theta$ on $(0,1)$. In fact,

$\varphi^{\prime}(\mu)=p \tau \mu^{\tau-\sigma-2}\left[(\tau-\sigma)(\mu-1)^{2}+\mu^{2}-1\right]+q \sigma$.

It is easy to verify that $\varphi^{\prime}(\mu)$ is strictly increasing on $(0,1)$. In addition,

$\varphi^{\prime}(\mu) \rightarrow-\infty \quad$ as $\quad \mu \rightarrow 0 \quad$ and $\quad \varphi^{\prime}(1)=q \sigma>0$.

Therefore, $\varphi^{\prime}(\mu)$ has a unique zero $\omega_{0}$ on $(0,1)$. Hence $\varphi(\mu)$ is strictly decreasing on $\left(0, \omega_{0}\right)$ and strictly increasing on $\left(\omega_{0}, 1\right)$. So that, $\varphi(\mu)$ has a unique zero $\theta$ on $(0,1)$ as $\varphi(\mu) \rightarrow+\infty(\mu \rightarrow 0)$ and $\varphi(1)=-q<0$.

Now, from (6), it follows that $g(\theta)$ is the maximum value of $g(\mu)$ on $(0,1)$. By (10), we know that (15) is equivalent to $g(\mu)<0$ for $\mu \in(0,1)$.

Theorem 2.13 Suppose that $p>1$ and $\tau<\sigma$. Then all the solutions of (1) are oscillatory if and only if $\theta<\bar{\theta}$, where $\theta$ and $\bar{\theta}$ are the unique zeros of $\varphi(\mu)$ and $h(\mu)$ on $(0,1)$, respectively.

Proof. Let

$y(\mu)=\frac{h(\mu)}{(\mu-1)^{2}}=q \mu^{\sigma+1}\left[\frac{\tau-\sigma}{\mu-1}+\frac{1}{(\mu-1)^{2}}\right]-\tau$,

then

$y^{\prime}(\mu)=\frac{q \mu^{\sigma}}{-(\mu-1)^{3}} z(\mu)$,

where

$z(\mu)=\sigma(\sigma-\tau)(\mu-1)^{2}-(2 \sigma-\tau)(\mu-1)+(\mu-1)+2$.

Further,

$z^{\prime}(\mu)=2 \sigma(\sigma-\tau)(\mu-1)-2 \sigma+\tau+1$.

It is easy to see that $z^{\prime}(\mu)$ is strictly increasing on $(0,1)$ and $z^{\prime}(1)=\tau-2 \sigma+1<0$. So that, $z(\mu)$ is strictly decreasing on $(0,1)$ and $z(\mu)>0$ on $(0,1)$ since $z(1)=2>0$. Consequently, $y(\mu)$ is strictly increasing on $(0,1)$. In addition,

$y(0)=-\tau \quad$ and $\quad y(\mu) \rightarrow+\infty\left(\mu \rightarrow 1^{-}\right)$.

Therefore, $y(\mu)$ and $h(\mu)$ have an equal and unique zero $\bar{\theta}$ on $(0,1)$. From Lemma 2.12, it is clear that (15) holds if and only if $\theta<\bar{\theta}$. 
Theorem 2.14 Assume that $p>1$ and $\tau<\sigma$. Then all the solutions of (1) are oscillatory if and only if there exists a real number $\alpha \in(0,1)$ such that

$\varphi(\alpha)=p \tau(\alpha-1)^{2} \alpha^{\tau-\sigma-1}+q[\sigma(\alpha-1)-1]<0$,

$h(\alpha)=q \alpha^{\sigma+1}[(\tau-\sigma)(\alpha-1)+1]-\tau(\alpha-1)^{2}<0$.

Proof. From the proof of Lemma $2.12, \varphi^{\prime}(\mu)$ has a unique zero $\omega_{0}$ on $(0,1)$, and $\varphi(\mu)$ is strictly decreasing on $\left(0, \omega_{0}\right)$ and strictly increasing on $\left(\omega_{0}, 1\right)$. Further,

$\varphi(\mu) \rightarrow+\infty(\mu \rightarrow 0)$ and $\varphi(1)=-q<0$.

Hence, $\varphi(\mu)$ has a unique zero $\theta$ on $(0,1)$.

Now, from the proof of Theorem $2.13, y(\mu)$ has a unique zero $\bar{\theta}$ on $(0,1)$ and is strictly increasing on $(0,1)$. Therefore, from Theorem 2.13, we obtain Theorem 2.14. The proof is complete.

Remark 2.15 The equation (1) has a non-oscillatory solution if and only if there exists a real number $\beta \in(0,1)$ such that

$\varphi(\beta)=p \tau(\beta-1)^{2} \beta^{\tau-\sigma-1}+q[\sigma(\beta-1)-1] \geq 0$,

$h(\beta)=q \beta^{\sigma+1}[(\tau-\sigma)(\beta-1)+1]-\tau(\beta-1)^{2} \geq 0$.

Furthermore, the function $\varphi(\mu)$ has a unique zero $\theta$ on $(0,1)$ and

$\varphi(\mu)>0$ for $\mu \in(0, \theta)$;

$\varphi(\mu)<0$ for $\mu \in(\theta, 1)$.

The function $h(\mu)$ has a unique zero $\bar{\theta}$ on $(0,1)$ and

$h(\mu)<0$ for $\quad \mu \in(0, \bar{\theta})$;

$h(\mu)>0$ for $\quad \mu \in(\bar{\theta}, 1)$.

Lemma 2.16 Let $p>1$ and $\tau=\sigma$. Then all the solutions of (1) are oscillatory if and only if

$q \theta^{\tau+1}<\tau(\theta-1)^{2}$,

where

$\theta=1-\left[\frac{q(\tau-1)+\sqrt{q^{2}(\tau-1)^{2}+4 q(p+q) \tau}}{2(p+q) \tau}\right]$.

By using similar procedure which used to prove Lemma 2.12, we can obtain the result. Therefore, we omit the proof.

Theorem 2.17 Suppose that $p>1$ and $\tau=\sigma$. Then all the solutions of (1) are oscillatory if and only if $\theta<\bar{\theta}$, where $\theta=1-\left[\frac{q(\tau-1)+\sqrt{q^{2}(\tau-1)^{2}+4 q(p+q) \tau}}{2(p+q) \tau}\right]$ and $\bar{\theta}$ is a unique zero of $h(\mu)=q \mu^{\tau-1}-\tau(\mu-1)^{2}$ on $(0,1)$.

Proof. From (8), we have that $h(\mu)=q \mu^{\tau+1}-\tau(\mu-1)^{2}$. Let

$y(\mu)=\frac{h(\mu)}{(\mu-1)^{2}}=\frac{q \mu^{\tau+1}}{(\mu-1)^{2}}-\tau$,

then

$y^{\prime}(\mu)=\frac{q \mu^{\tau}}{-(\mu-1)^{3}} z(\mu)$,

where

$z(\mu)=-(\tau+1)(\mu-1)+2(\mu-1)+2$.

Since $z(1)=2>0$, we have that $z(\mu)>0$ for $\mu \in(0,1)$. This means that $y^{\prime}(\mu)>0$ for $\mu \in(0,1)$. Hence $y(\mu)$ is strictly increasing on $(0,1)$. In addition, $y(0)=-\tau$ and $y(\mu) \rightarrow+\infty\left(\mu \rightarrow 1^{-}\right)$.

In consequence, the function $y(\mu)$ has a unique zero $\bar{\theta}$ on $(0,1)$, which implies together with Lemma 2.16 that $q \theta^{\tau+1}<\tau(\theta-1)^{2}$ if and only if $\theta<\bar{\theta}$. 
Theorem 2.18 Assume that $p>1$ and $\tau=\sigma$. Then all the solutions of (1) are oscillatory if and only if there exists a positive real number $\alpha \in[\theta, 1)$ such that

$h(\alpha)=q \alpha^{\tau+1}-\tau(\alpha-1)^{2}<0$,

where

$\theta=1-\left[\frac{q(\tau-1)+\sqrt{q^{2}(\tau-1)^{2}+4 q \tau(p+q)}}{2(p+q) \tau}\right]$.

Proof. From the proof of Theorem 2.17, the function $y(\mu)$ has a unique zero $\bar{\theta}$ on $(0,1)$ and is strictly increasing on $(0,1)$. Now, it is easy to find that the signs of the functions $h(\mu)$ and $y(\mu)$ are the same in the interval $(0,1)$. So that, from Theorem 2.17, we obtain Theorem 2.18 immediately. The proof is complete.

Remark 2.19 The function $h(\mu)$ has a unique zero $\bar{\theta}$ on $(0,1)$ and

$h(\mu)<0 \quad$ for $\quad \mu \in(0, \bar{\theta})$;

$h(\mu)>0$ for $\mu \in(\bar{\theta}, 1)$.

Corollary 2.20 If $p>1, \tau=\sigma$ and $q \geq \frac{p^{\tau+1}}{\tau(p+q)^{\tau-1}}$, then all the solutions of (1) are oscillatory.

Proof. Let $\alpha=\frac{p}{p+q}$, then

$\theta=1-\left[\frac{q(\tau-1)+\sqrt{q^{2}(\tau-1)^{2}+4 q \tau(p+q)}}{2(p+q) \tau}\right]<\alpha<1$.

It follows that

$h(\alpha)=q \alpha^{\tau+1}-\tau(\alpha-1)^{2}=\frac{q}{(p+q)^{2}}\left[\frac{p^{\tau+1}}{(p+q)^{\tau-1}}-q \tau\right] \leq 0$.

From Theorem 2.18, it can be concluded that all the solutions of (1) are oscillatory.

Theorem 2.21 Suppose that $p>1, \tau<\sigma$ and

$\xi=1+\left[\frac{\tau+1-2 \sigma-\sqrt{(2 \sigma-\tau-1)^{2}+8 \sigma(\tau-\sigma)}}{2 \sigma(\tau-\sigma)}\right]$.

Then all the solutions of (1) are oscillatory if and only if one of the following conditions holds.

$\left(\mathrm{H}_{1}\right) \quad q \geq \frac{p \tau \xi^{\tau-\sigma-1}(\xi-1)^{2}}{1-\sigma(\xi-1)} ;$

$\left(\mathrm{H}_{2}\right) h(\theta)=q \theta^{\sigma+1}[(\tau-\sigma)(\theta-1)+1]-\tau(\theta-1)^{2}<0$, where $\theta$ is a unique zero of $(7)$ on $(\xi, 1)$.

Proof. Let

$\phi(\mu)=\frac{\varphi(\mu)}{1-\sigma(\mu-1)}=\frac{p \tau \mu^{\tau-\sigma-1}(\mu-1)^{2}}{1-\sigma(\mu-1)}-q$,

then by (6) and (7), we have that

$\varphi(\mu)=[1-\sigma(\mu-1)] \phi(\mu)$,

$g^{\prime}(\mu)=\frac{\mu^{\sigma}[1-\sigma(\mu-1)] \phi(\mu)}{(\mu-1)^{2}}$.

Differentiation yields that

$\phi^{\prime}(\mu)=\frac{-p \tau \mu^{\tau-\sigma-2}(\mu-1)}{[1-\sigma(\mu-1)]^{2}} y(\mu)$, 
where $y(\mu)=\sigma(\tau-\sigma)(\mu-1)^{2}+(2 \sigma-\tau-1)(\mu-1)-2$ and $y(\mu)$ satisfies that $y(0) \geq 0$ and $y(1)=-2<0$. Thus, $y(\mu)$ has a unique zero

$\xi=1+\left[\frac{\tau+1-2 \sigma-\sqrt{(2 \sigma-\tau-1)^{2}+8 \sigma(\tau-\sigma)}}{2 \sigma(\tau-\sigma)}\right] \quad$ on $\quad(0,1)$

Consequently, $\phi^{\prime}(\mu)>0$ and $\phi^{\prime}(\mu)<0$ for $\mu \in(0, \xi)$ and $\mu \in(\xi, 1)$, respectively. As a result, the function $\phi(\mu)$ is strictly increasing on $(0, \xi)$ and strictly decreasing on $(\xi, 1)$. This implies that $\phi(\xi)$ is the maximum value of $\phi(\mu)$ for $\mu \in(0,1)$. In addition,

$\phi(0)=\phi(1)=-q$

So that, if $q \geq \frac{p \tau \xi^{\tau-\sigma-1}(\xi-1)^{2}}{1-\sigma(\xi-1)}$, i.e. $\phi(\xi) \leq 0$, then we have that $g^{\prime}(\mu) \leq 0, \mu \in(0,1)$. Now, it is easy to find that $g(0)=-1$. Hence, (14) holds.

If $q<\frac{p \tau \xi^{\tau-\sigma-1}(\xi-1)^{2}}{1-\sigma(\xi-1)}$, i.e. $\phi(\xi)>0$, consequently, $\phi(\mu)$ has a unique zero $\omega$ on $(0, \xi)$ and a unique zero $\theta$ on $(\xi, 1)$. In consequence, we have that $g^{\prime}(\mu)<0$ on $(0, \omega), g^{\prime}(\mu)>0$ on $(\omega, \theta)$ and $g^{\prime}(\mu)<0$ on $(\theta, 1)$, which means that the function $g(\mu)$ is strictly decreasing, strictly increasing and strictly decreasing on $(0, \omega),(\omega, \theta)$ and $(\theta, 1)$, respectively. Further,

$g(0)=-1$ and $\lim _{\mu \rightarrow 1^{-}} g(\mu)=-\infty$

and $\phi(\theta)=0$, i.e. $\varphi(\theta)=0$. Therefore, (14) holds if and only if $g(\theta)<0$ (the condition $\left(H_{2}\right)$ holds).

Theorem 2.22 Suppose that $p>1, \tau>\sigma$ and $q<\frac{p \tau \xi^{\tau-\sigma-1}(\xi-1)^{2}}{1-\sigma(\xi-1)}$. Then all the solutions of (1) are oscillatory if and only if $\theta<\bar{\theta}$, where $\theta$ and $\bar{\theta}$ are the unique zeros of (7) and (8) on $(\xi, 1)$, respectively and

$\xi=1+\left[\frac{\tau-2 \sigma+1-\sqrt{(2 \sigma-\tau-1)^{2}+8 \sigma(\tau-\sigma)}}{2 \sigma(\tau-\sigma)}\right]$.

Proof. Let

$y(\mu)=\frac{h(\mu)}{(\mu-1)^{2}}=q \mu^{\sigma+1}\left(\frac{\tau-\sigma}{\mu-1}+\frac{1}{(\mu-1)^{2}}\right)-\tau$,

then

$y^{\prime}(\mu)=\frac{q \mu^{\sigma}}{-(\mu-1)^{3}} z(\mu)$,

where

$z(\mu)=-\sigma(\tau-\sigma)(\mu-1)^{2}-(2 \sigma-\tau-1)(\mu-1)+2$

and $z(\mu)$ satisfies that $z(0) \leq 0$ and $z(1)=2>0$. Therefore, $z(\mu)$ has a unique zero

$\omega=1+\left[\frac{\tau-2 \sigma+1-\sqrt{(2 \sigma-\tau-1)^{2}+8 \sigma(\tau-\sigma)}}{2 \sigma(\tau-\sigma)}\right]$ on $(0,1)$.

This means that $z(\mu)<0$ for $\mu \in(0, \omega)$ and $z(\mu)>0$ for $\mu \in(\omega, 1)$. Consequently, $y^{\prime}(\mu)<0$ and $y^{\prime}(\mu)>0$ for $\mu \in(0, \omega)$ and $y \in(\omega, 1)$, respectively. In consequence, $y(\mu)$ is strictly decreasing on $(0, \omega)$ and strictly increasing on $(\omega, 1)$. In addition,

$y(0)=-\tau \quad$ and $\quad \lim _{\mu \rightarrow 1^{-}} y(\mu)=+\infty$.

Thus, $y(\mu)$ has a unique zero $\bar{\theta}$ on $(\omega, 1)$. It is easy to find that $\omega=\xi$. This, together with Theorem 2.21 imply that $h(\theta)<0$ if and only if $\theta<\bar{\theta}$. 
Theorem 2.23 Assume that $p>1, \tau>\sigma$ and

$\xi=1+\left[\frac{\tau-2 \sigma+1-\sqrt{(2 \sigma-\tau-1)^{2}+8 \sigma(\tau-\sigma)}}{2 \sigma(\tau-\sigma)}\right]$.

Then all the solutions of (1) are oscillatory if and only if there exists a real number $\alpha \in(\xi, 1)$ such that

$\varphi(\alpha)=p \tau(\alpha-1)^{2} \alpha^{\tau-\sigma-1}+q[\sigma(\alpha-1)-1]<0$,

$h(\alpha)=q \alpha^{\sigma+1}[(\tau-\sigma)(\alpha-1)+1]-\tau(\alpha-1)^{2}<0$.

Proof. From the proof of Theorem 2.22, $y(\mu)$ has a unique zero $\bar{\theta}$ on $(\xi, 1)$ and $y(\mu)$ is strictly increasing on $(\xi, 1)$. If $q \geq \frac{p \tau(\xi-1)^{2} \xi^{\tau-\sigma-1}}{1-\sigma(\xi-1)}$, then by Theorem 2.21, all the solutions of (1) are oscillatory. Now, again from the proof of Theorem 2.21, it follows that $\varphi(\mu)<0, \mu \in(\xi, 1)$. As a result, the condition of Theorem 2.23 hold.

If $q<\frac{p \tau(\xi-1)^{2} \xi^{\tau-\sigma-1}}{1-\sigma(\xi-1)}$, then from the proof of Theorem 2.21, the function $\phi(\mu)$ has a unique zero $\theta$ on $(\xi, 1)$ and is strictly decreasing on $(\xi, 0)$. Moreover, the function values of $\varphi(\mu)$ and $\phi(\mu)$ have the same sign in the interval $(\xi, 1)$. And also both functions $h(\mu)$ and $y(\mu)$ have the same sign in the interval $(\xi, 1)$. Therefore, from Theorem 2.22 , it can be seen that $\theta<\bar{\theta}$ if and only if the conditions of Theorems 2.23 hold. The proof is complete.

Remark 2.24 The function $h(\mu)$ has a unique zero $\bar{\theta}$ on $(\xi, 1)$ and

$h(\mu)<0$ for $(\xi, \bar{\theta})$;

$h(\mu)>0$ for $(\bar{\theta}, 1)$.

Further, we have that $q \geq \frac{p \tau(\xi-1)^{2} \xi^{\tau-\sigma-1}}{1-\sigma(\xi-1)}$ is equivalent to $\varphi(\mu)<0$ for $\mu \in(\xi, 1), q<\frac{p \tau(\xi-1)^{2} \xi^{\tau-\sigma-1}}{1-\sigma(\xi-1)}$ is equivalent to the fact that $\varphi(\mu)$ has a unique zero $\theta$ on $(\xi, 1)$. Here,

$\varphi(\mu)>0$ for $\mu \in(\xi, \theta)$;

$\varphi(\mu)<0$ for $\mu \in(\theta, 1)$.

Thus, (1) has a non-oscillatory solution if and only if there exists a real number $\beta \in(\xi, 1)$ such that

$\varphi(\beta)=p \tau(\beta-1)^{2} \beta^{\tau-\sigma-1}+q[\sigma(\beta-1)-1] \geq 0$,

$h(\beta)=q \beta^{\sigma+1}[(\tau-\sigma)(\beta-1)+1]-\tau(\beta-1)^{2} \geq 0$.

For $\tau>\sigma$, it is easy to verify that

$1-\frac{2}{\tau-\sigma}<\xi=1+\left[\frac{\tau-2 \sigma+1-\sqrt{(2 \sigma-\tau-1)^{2}+8 \sigma(\tau-\sigma)}}{2 \sigma(\tau-\sigma)}\right] \leq 1-\frac{1}{\tau-\sigma}$.

From the condition $\left(H_{1}\right)$ of Theorem 2.21, we obtain the following corollary at once.

Corollary 2.25 If $p>1, \tau>\sigma$ and $q \geq \frac{4 p}{\tau-\sigma}$, then all the solutions of (1) are oscillatory.

In fact, Corollary 2.25 can be still improved and extended, we have given the following corollary to Corollary 2.25 with some improvements.

Corollary 2.26 If $p>1, \tau>\sigma$ and $q \geq \frac{p(\tau-\sigma-1)^{\tau-\sigma-1}}{(\tau-\sigma)^{\tau-\sigma}}$, then all the solutions of (1) are oscillatory.

Proof. It is easy to see that

$\xi=1+\left[\frac{\tau-2 \sigma+1-\sqrt{(2 \sigma-\tau-1)^{2}+8 \sigma(\tau-\sigma)}}{2 \sigma(\tau-\sigma)}\right] \leq \alpha<1$,

where $\alpha=1-\frac{1}{\tau-\sigma}$. In consequence,

$\varphi(\alpha)=p \tau(\alpha-1)^{2} \alpha^{\tau-\sigma-1}+q[\sigma(\alpha-1)-1]$

$=\frac{\tau}{(\tau-\sigma)^{2}}\left[p\left(\frac{\tau-\sigma-1}{\tau-\sigma}\right)^{\tau-\sigma-1}-q(\tau-\sigma)\right] \leq 0$,

$h(\alpha)=q \alpha^{\sigma+1}[(\tau-\sigma)(\alpha-1)+1]-\tau(\alpha-1)^{2}=\frac{-\tau}{(\tau-\sigma)^{2}}<0$.

From Theorem 2.23, all the solutions of (1) are oscillatory. 


\section{Some examples}

In this section, we enumerate some specific examples to verify the results that we obtained and to show the simplicity of the results.

Example 3.1 Consider the first order neutral delay difference equation

$\Delta\left[x(n)-\frac{1}{2} x(n-2)\right]+x(n-4)=0$.

Then, it is easy to see that $p=\frac{1}{2}, q=1, \tau=2$ and $\sigma=4$. Consequently, $\tau<\sigma$, and $p \in(0,1)$. Also

$\theta=1+\left[\frac{-\frac{q(\sigma+1)^{\sigma+1}}{\sigma^{\sigma}}+\sqrt{q\left(\frac{(\sigma+1)^{\sigma+1}}{\sigma^{\sigma}}\right)^{2}+4 p q \tau\left(\frac{\sigma+1}{\sigma}\right)^{\sigma+1}}}{2 p \tau}\right]$

$=1.049019$

Let $\alpha=1.1$. Then $\alpha \in\left[\theta, 1+\frac{1}{\sigma}\right)$ and $q \alpha^{\sigma+1} \geq \sigma(\alpha-1)^{2}$. Corollary 2.7 shows that all the solutions of (18) are oscillatory. One such solution is $x(n)=(-1)^{n}$.

Example 3.2 Consider the first order neutral delay difference equation

$\Delta\left[x(n)-\frac{1}{32} x(n-4)\right]+\frac{3}{16} x(n-2)=0$.

Here, $p=\frac{1}{32}, q=\frac{3}{16}, \tau=4$ and $\sigma=2$. Consequently, $\tau>\sigma$ and $q>\frac{p(\tau-\sigma-1)^{\tau-\sigma-1}}{(\tau-\sigma)^{\tau-\sigma}}$. Then by Corollary 2.26, all the solutions of (19) are oscillatory. One such solution is $x(n)=\left(\frac{-1}{2}\right)^{n}$.

Example 3.3 Consider the first order neutral delay difference equation

$\Delta\left[x(n)-\frac{1}{9} x(n-4)\right]+\frac{32}{81} x(n-3)=0$.

Then, it is easy to see that $p=\frac{1}{9}, q=\frac{32}{81}, \tau=4$ and $\sigma=3$. Consequently, $\tau>\sigma$ and

$\theta=1+\left[\frac{-q \sigma+\sqrt{(q \sigma)^{2}+4 p q \tau}}{2 p \tau}\right]=1.2996$

If we take $\alpha=1.31$, then $\alpha \in\left[\theta, 1+\frac{1}{\sigma}\right)$ and $q \alpha^{\sigma+1}-\tau(\alpha-1)^{2}>0$. From Corollary 2.9, it is easy to conclude that all the solutions of (20) are oscillatory. One such solution is $x(n)=\left(\frac{-1}{3}\right)^{n}$.

Example 3.4 Consider the first order neutral delay difference equation

$\Delta[x(n)-10 x(n-4)]+18 x(n-4)=0 . \quad n \geq 0$

Then, it is easy to see that $p=10, q=18, \tau=4$ and $\sigma=4$. Consequently, $\tau=\sigma$ and $q>\frac{p^{\tau+1}}{\tau(p+q)^{\tau-1}}$. Corollary 2.20 shows that all the solutions of $(21)$ are oscillatory. One such solution is $x(n)=(-2)^{n}$.

\section{References}

[1] R. P. Agarwal, Difference Equations and Inequalities: Theory, Methods and Applications, Marcel Dekker, New York, 1999.

[2] R.P. Agarwal and P. J. Y. Wong, Advanced Topics in Difference Equations, Kluwer, Dodrecht, 1997.

[3] M. P. Chen, B. S. Lalli and J. S. Yu, Oscillation in neutral delay difference equations with variable coefficients, Comput. Math. Appl. 29(3) (1995), 5-11. 
[4] S. N. Elaydi, An Introduction to Difference Equations, Springer Verlag, New York, 1996.

[5] Ethiraju Thandapani, Ramalingam Arul and Palanisamy S. Raja, Oscillation of first order neutral delay difference equations, Appl. Math. E-Notes, 3(2003), 88-94.

[6] D. A. Georgiou, E. A. Grove and G. Ladas, Oscillations of neutral difference equations, Appl. Anal. 33(1989), $243-253$.

[7] I. Gyori and G. Ladas, Oscillation Theory of Delay Differential Equations with Applications, Clarendon Press, Oxford, 1991.

[8] W. G. Kelley and A. C. Peterson, Difference Equations: An introduction with Applications, Clarendon Press, Oxford 1991.

[9] V. Lakshmikantham and D. Trigiante, Theory of Difference Equations, Academic Press, New york, 1988.

[10] B. S. Lalli and B. G. Zhang, On existence of positive solutions and bounded oscillations for neutral difference equations, J. Math. Anal. Appl. 166(1992), 272-287.

[11] B. S. Lalli and B. G. Zhang, Oscillation and comparison theorems for certain neutral difference equations, J. Austral. Math. Soc. Ser. B34(1992), 245-256.

[12] G. Ladas, Explicit conditions for the oscillation of difference equations, J. Math. Anal. Appl. 153(1990), $276-287$.

[13] Özkan Öcalan, Oscillation criteria for systems of difference equations with variable coefficients, Appl. Math. E-Notes, 6(2006), 119-125.

[14] X. H. Tang and Xiaoyan Lin, Necessary and sufficient conditions for oscillation of first - order nonlinear neutral difference equations, Comput. Math. Appl. 55(2008), 1279-1292.

[15] Xiaohui Gong, Xiaozhu zhong, Jianqiang Jia, Rui Ouyang and Hongqiang Han, Oscillation of first order Neutral Difference Equation, Modern Applied Science, 3(8) (2009), 90-94.

[16] Ying Gao and Guang Zhang, Oscillation of nonlinear first order neutral difference equations, Appl. Math. E-Notes, $1(2001), 5-10$.

[17] Z. Zhou and J. S. Yu, Linearized oscillations for difference equations of neutral type, Math. Sci. Res. Hot-Line $1(11)(1997), 1-8$. 